\title{
PERFIL DE MÃES E O DESFECHO DO NASCIMENTO PREMATURO OU A TERMO*
}

\author{
Gracimary Alves Teixeira ${ }^{1}$, Jovanka Bittencourt Leite de Carvalho², Brismark Góes da Rocha ${ }^{3}$, Silvana Alves \\ Pereira ${ }^{4}$, Bertha Cruz Enders ${ }^{5}$
}

RESUMO: Objetivo: identificar o perfil de mães de bebês prematuros e a termo para o desfecho do nascimento. Método: trata-se de um estudo analítico-descritivo, seccional, com amostra de 244 puérperas, sendo 109 mães de bebês prematuros e 135 mães de bebês a termo, por partos ocorridos no período de abril a setembro de 2015, em uma maternidade pública do nordeste brasileiro. Os dados foram analisados pelo programa Statistica 10 e teste Qui-quadrado. Resultados: alta prevalência de sedentarismo; significância estatística para sobrepeso e obesidade antes e durante a gravidez; elevada prevalência de pressão arterial alta durante a gestação das mães de bebês prematuros. Logo, os bebês nasceram com problemas de saúde em $58,1 \%(n=63)$ dos prematuros e nascimento saudável em $96,3 \%(n=130)$ dos bebês a termo. Conclusão: o perfil de mães com obesidade e sobrepeso é fator de risco ao nascimento do bebê prematuro como evento que demanda cuidado à saúde da criança.

DESCRITORES: Prematuro; Cuidado Pré-Natal; Parto; Fatores de Risco; Enfermagem Materno-Infantil.

\section{IMPACT OF MATERNAL PROFILE ON BIRTH OUTCOMES}

\begin{abstract}
Objective: to identify the impact of maternal profile on birth outcomes. Method: Descriptive cross-sectional study with a sample of 244 postpartum women, as follows: 109 mothers of preterm infants and 135 mothers of full-term infants. The births occurred in the April-September 2015 period in a public maternity hospital in the Northeastern region of Brazil. Data was analyzed with Statistica 10 software and Chi-square test. Results: high prevalence of sedentary lifestyle; statistical significance for overweight and obesity before and during pregnancy; high prevalence of high blood pressure during the gestational period of mothers of preterm infants. Also, $58.1 \%(n=63)$ of preterm infants were born with health problems and $96.3 \%(n=130)$ of full-term infants were healthy. Conclusion: Maternal obesity and overweight is a risk factor for preterm births, an event that demands child healthcare.

DESCRIPTORS: Preterm; Prenatal care; Childbirth; Risk Factors; Maternal-Child Nursing.
\end{abstract}

\section{PERFIL DE MADRES Y EL DESENLACE DEL NACIMIENTO PREMATURO O A TÉRMINO}

RESUMEN: Objetivo: identificar el perfil de madres de bebés prematuros y a término para el desenlace del nacimiento. Método: es un estudio analítico descriptivo, seccional, con muestra de 244 puérperas, siendo 109 madres de bebés prematuros y 135 madres de bebés a término, por partos ocurridos el periodo de abril a septiembre de 2015, en una maternidad pública de nordeste de Brasil. Se analizaron los datos por medio del programa Statistica 10 y test Chi-cuadrado. Resultados: hubo grande prevalencia de sedentarismo; significancia estadística para sobrepeso y obesidad antes y durante la gravidez; alta prevalencia de presión arterial alta durante la gestación de las madres de bebés prematuros. De ese modo, 58,1\% $(n=63)$ de los bebés prematuros nacieron con problemas de salud y de los bebés a término hubo nacimiento saludable en $96,3 \%(n=130)$. Conclusión: el perfil de madres con obesidad y sobrepeso es factor de riesgo para el nacimiento del bebé prematuro y eso demanda cuidado a la salud del niño.

DESCRIPTORES: Prematuro; Cuidado Prenatal; Parto; Factores de Riesgo; Enfermería Materno Infantil.

*Artigo extraído da dissertação intitulada: "Perfil de mães e o desfecho do nascimento prematuro ou termo". Universidade Federal do Rio Grande do Norte, 2015.

${ }^{1}$ Enfermeira. Doutoranda em Enfermagem. Docente da Universidade Federal do Rio Grande do Norte. Natal, RN, Brasil. ${ }^{2}$ Enfermeira. Doutora em Ciências da Saúde. Docente do Programa de Pós-graduação em Enfermagem da Universidade Federal do Rio Grande do Norte. Natal, RN, Brasil.

${ }^{3}$ Estatístico. Doutor em Engenharia Mecânica. Docente da Universidade Estadual do Rio Grande do Norte. Natal, RN, Brasil. ${ }^{4}$ Fisioterapeuta. Doutora em Neurociências e Comportamento. Docente da Universidade Federal do Rio Grande do Norte. Natal, RN, Brasil.

${ }^{5}$ Enfermeira. Doutora em Enfermagem. Docente do Programa de Pós-graduação em Enfermagem da Universidade Federal do Rio Grande do Norte. Natal, RN, Brasil. 


\section{INTRODUÇÃO}

O nascimento do bebê prematuro (com menos de 37 semanas de gestação) é considerado problema de saúde pública mundial, pois é tido como um dos principais fatores de risco para morbidade e mortalidade neonatal ${ }^{(1)}$, causa repercussões clínicas que demandam cuidados de maior complexidade e, às vezes, por toda a vida, acarretando alto custo social e econômico aos países, visto que o nascimento prematuro resulta de um conjunto de fatores inter-relacionados, os quais impactam na qualidade de vida dos que sobrevivem com sequelas ou na mortalidade infantil ${ }^{(2)}$.

Em 2014, no ranking mundial da Taxa de Mortalidade Infantil (TMI), os primeiros lugares foram ocupados por nações com índices de mortalidade muito elevados, como o Afeganistão ( $1^{\circ}$ lugar) com 117,23 por mil nascidos vivos; Mali ( $2^{\circ}$ lugar) com 104,34 por mil nascidos vivos; e Somália ( $3^{\circ}$ lugar) com 100,14 por mil nascidos vivos. Já os países com menor TMI foram Mônaco (1,81 por mil nascidos vivos), Japão (2,13 por mil nascidos vivos) e Bermudas (2,48 por mil nascidos vivos) ${ }^{(3)}$.

No Brasil, a taxa de mortalidade neonatal ( 0 a 27 dias de vida) é considerada o principal componente da TMI, principalmente o neonato precoce ( 0 a 6 dias de vida) devido à persistência dos problemas na preconcepção ou durante a gestação. Essa TMI diminuiu de 26,6 para 16,2 por mil nascidos vivos entre os anos de 2000 e 2010 em virtude da importante queda na mortalidade pós-neonatal (27 dias a 1 ano de vida) em razão das ações centradas na prevenção e no tratamento de infecções. Nesse mesmo período, a TMI na região Nordeste reduziu de 38,4 para 20,1 por mil nascidos vivos e, destes, a taxa de mortalidade neonatal era de 22,7 e reduziu para 14,3. Dessa forma, o período pós-neonatal apresentou, em 2010, a taxa de mortalidade de 5,8, enquanto o neonatal com 14,3, caracterizando-se como a faixa de concentração da mortalidade infantil ${ }^{(4)}$.

As complicações do parto pré-termo foram uma das principais causas de mortes neonatais globais em 2008 e o número de crianças pré-termo e com baixo peso ao nascer vem aumentando constantemente durante as últimas décadas ${ }^{(5)}$. A prematuridade foi responsável por $14 \%$ das causas de mortes de crianças no mundo ${ }^{(6)}$. Globalmente, $11,1 \%$ dos bebês nascem pré-termo, o que equivale a mais de 15 milhões de crianças ou mais de 1 em cada 10 crianças, em que mais de $60 \%$ desses nascimentos ocorrem na África e sul da Ásia, no entanto os 10 países com as maiores taxas incluem o Brasil, os EUA, a Índia e a Nigéria ${ }^{(7)}$.

Considerando o perfil de mães para o nascimento de bebês prematuros e a termo, há a incógnita no meio científico. O parto pré-termo resulta de fatores inter-relacionados em que os estudos apontam associação com problemas na preconcepção ou durante a gestação, aspectos ambientais e sociais, e altos índices de cesáreas sem indicação antes da maturidade fetal ${ }^{(2,8-10)}$.

Assim, a teorização que embasa esta pesquisa é a teoria de risco, sendo o termo risco definido como "probabilidade de um membro de uma população definida desenvolver uma dada doença em um período de tempo". A partir desse conceito, a epidemiologia busca a associação de determinados fatores de risco com as patologias ${ }^{(11)}$. Diante do exposto, este estudo objetiva identificar o perfil de mães e o desfecho do nascimento de bebês prematuros e a termo.

Nesse contexto, conforme o decreto $\mathrm{n}^{\circ}$ 94.406/87 que regulamenta a lei do exercício profissional da enfermagem de $\mathrm{n}^{\circ} 7.498 / 86$, o enfermeiro tem papel fundamental na saúde materno-infantil, tendo em vista o acompanhamento da gestante de risco habitual e puérpera através de consultas de pré-natal e puerperal ${ }^{(12)}$. E em caso de pré-natal de alto risco, participa juntamente com outros profissionais, constituindo equipe multidisciplinar. Além disso, o enfermeiro obstetra presta assistência ao parto de evolução fisiológica (sem distócia) e ao recém-nascido, conforme a resolução do COFEN nº 0478/2015 ${ }^{(13)}$.

\section{- MÉTODO}

Trata-se de um estudo analítico-descritivo, seccional de cunho quantitativo, realizado em uma maternidade pública, localizada na região Nordeste do Brasil, estado do Rio Grande do Norte.

Essa maternidade presta assistência pré-natal às gestantes de alto risco, referenciadas pela atenção 
primária à saúde. Além disso, dispõe de classificação de risco, centro obstétrico (pré-parto e parto), centro cirúrgico, alojamento conjunto, médio risco para recém-nascidos, unidade de tratamento clínico para gestantes e recém-nascidos, Unidade de Terapia Intensiva Neonatal, banco de leite, serviço de ultrassonografia, central de material de esterilização.

A escolha dessa maternidade para estudo se deu em virtude de o município apresentar estruturação em rede de atenção à saúde materno-infantil, com ações e serviços articulados em níveis de complexidade crescente. Além disso, em 2012, o município apontou que 78,94\% dos óbitos neonatais na primeira semana de vida foram de recém-nascidos pré-termo e ainda apresentaram prevalência de nascidos vivos pré-termo de 13,20\%, ambas as prevalências superiores à nacional ${ }^{(14)}$.

A população foi composta por 1508 mães de nascidos por parto hospitalar, no período de abril a setembro de 2015, dessas foi selecionada a amostra de 244 puérperas, sendo formados dois grupos. Um constituído por 109 mães de bebês prematuros registrados nos livros da sala de parto e centro obstétrico, pois das 155 mães de bebês prematuros registrados no período em estudo, 35 foram eliminadas por incompatibilidade e incertezas da Idade Gestacional (IG), também ocorreram perdas de quatro mães por transferência para outra maternidade e sete por alta hospitalar antes de $48 \mathrm{~h}$ de pós-parto, o que impossibilitou o contato.

O segundo grupo foi formado por cálculo amostral obtido como base na média dos partos entre os anos de 2012 e 2013, no período em apreço, resultando em 135 mães de bebês nascidos a termo selecionados de forma aleatória, pelo sorteio a cada 10 mães registradas no livro de parto uma era entrevistada.

Para a definição de pré-termo e a termo, considerou-se a IG calculada pela Data da Última Menstruação (DUM) relatada pela mãe que não tinha dúvida, pela ultrassonografia obstétrica realizada com menos de 20 semanas e registro da IG por pediatra ou obstetra.

Para inclusão, foram considerados: a) residir no município de Parnamirim-RN; b) ter realizado acompanhamento pré-natal em Unidades de Saúde da Família de Parnamirim ou planos de saúde/ privados de Parnamirim ou não ter nenhuma consulta de pré-natal; c) parto hospitalar no Hospital Materno Infantil Divino Amor. Foram excluídos todos os recém-nascidos que não tiverem IG registrada ou a IG era divergente, acrescido da situação de possíveis doenças maternas psíquicas que revelassem o grau de vulnerabilidade e risco da mãe, advindos do prejuízo cognitivo na coleta de dados.

Para coleta de dados, foram utilizados quatro instrumentos: 1) livro de registros da sala de parto e centro obstétrico do Hospital Materno Infantil Divino Amor; 2) livro de ocorrência da enfermagem com os internamentos; 3 ) coleta de dados das informações existentes nos documentos: cartão e prontuário da gestante; 4) formulário de entrevista adaptado(13).

A entrevista com as mães ocorreu durante sua permanência na maternidade, em ambiente privativo e após a assinatura do Termo de Consentimento Livre e Esclarecido. Parte deste estudo foi retrospectivo, as informações dos registros foram confirmadas com as lembranças/memória das puérperas, com isso não houve perdas por falta de registros.

Após compilado os dados, avaliou-se as estatísticas analítico-descritivas através do Statística 10, pelo qual foram identificadas as frequências, proporções e, por fim, considerou-se o nível de significância $\mathrm{p}<0,05 \%$ para o teste Qui-quadrado.

Os parâmetros de normalidade utilizado na pesquisa quanto ao peso e estado nutricional da mãe e do bebê, pressão arterial da gestante, índice de apgar, foram definidos como:

- O estado nutricional pré-gestacional da mãe foi calculado pelo Índice de Massa Corporal (IMC) compreendido pela divisão entre o peso e altura ao quadrado. IMC normal (eutrófico): $\geq 20 \mathrm{~kg} / \mathrm{m}^{2} \mathrm{e}<25$ $\mathrm{kg} / \mathrm{m}^{2}$; IMC sobrepeso: $\geq 25 \mathrm{~kg} / \mathrm{m}^{2}$ e $<30 \mathrm{~kg} / \mathrm{m}^{2} ;$ IMC obesidade: $\geq 30 \mathrm{~kg} / \mathrm{m}^{2}$. Durante a gestação também foi utilizado o IMC, porém de acordo com a Idade Gestacional (IG). Os níveis de pressão arterial considerado normal foi o de $<140 \times 90 \mathrm{mmHg}^{(15)}$.

- O baixo peso ao nascer definido pela OMS como peso menor que $2500 \mathrm{~g}$ no momento do nascimento ${ }^{(16)}$. O índice de Apgar, preconizado pela OMS. Avalia-se frequência cardíaca, respiratória, tônus muscular, irritabilidade reflexa e coloração da pele, no primeiro e quinto minuto após o 
nascimento, tendo como classificação sem asfixia de 8 a 10, asfixia leve de 5 a 7, asfixia moderada 3 a 4 e asfixia grave 2 a $0^{(16)}$.

O projeto foi submetido ao Comitê de Ética e Pesquisa da Universidade Federal do Rio Grande do Norte para fins de apreciação, com parecer favorável de nº 1.047.431/15.

\section{RESULTADOS}

Os resultados apresentam o perfil socioeconômico, características e condições de mães de bebês prematuros e a termo, durante a gestação, para o desfecho do nascimento.

Na Tabela 1, identificou-se que o perfil socioeconômico das mães de bebês prematuros e a termo apresenta baixo nível de escolaridade, renda familiar aquém do salário mínimo vigente no país, além de moradia inadequada com 2 a 3 cômodos. E ainda mais agravante, a situação das mães de bebês prematuros com alta prevalência de moradia em imóvel alugado e com gravidez na faixa etária da adolescência (Tabela 2).

Tabela 1 - Condições socioeconômicas das mães de bebês prematuros e a termo. Parnamirim, RN, Brasil, 2015 (continua)

\begin{tabular}{|c|c|c|c|c|}
\hline \multirow[t]{2}{*}{ Variáveis } & \multicolumn{2}{|c|}{ Pré-termo } & \multicolumn{2}{|c|}{ Termo } \\
\hline & $\%$ & $\mathbf{f}$ & $\%$ & $f$ \\
\hline Idade $<20$ anos & 16,51 & 8 & 2,96 & 37 \\
\hline 20 a 35 anos & 76,15 & 83 & 85,19 & 82 \\
\hline$\geq 36$ anos & 7,34 & 8 & 11,85 & 16 \\
\hline \multicolumn{5}{|l|}{ Tipo de união } \\
\hline Casado/mora junto & 85,32 & 93 & 88,89 & 120 \\
\hline Solteira & 11,93 & 13 & 8,89 & 12 \\
\hline Outros & 2,75 & 3 & 2,22 & 3 \\
\hline \multicolumn{5}{|l|}{ Escolaridade } \\
\hline Fundamental incompleto & 22,02 & 24 & 26,67 & 36 \\
\hline Médio completo & 33,94 & 37 & 31,85 & 43 \\
\hline Nível superior & 9,17 & 10 & 6,67 & 9 \\
\hline \multicolumn{5}{|l|}{ Renda } \\
\hline$<1$ salário mínimo & 11,93 & 13 & 7,41 & 10 \\
\hline Entre 1 e 2 salários & 64,22 & 70 & 71,85 & 97 \\
\hline Entre 3 e 5 salários & 21,1 & 23 & 19,26 & 26 \\
\hline$\geq 6$ salários & 2,75 & 3 & 1,48 & 2 \\
\hline \multicolumn{5}{|l|}{ Número de filhos } \\
\hline 3 filhos & 36,7 & 40 & 37,4 & 50 \\
\hline 4 filhos & 31,19 & 34 & 28,89 & 39 \\
\hline 5 filhos & 20,18 & 22 & 19,26 & 26 \\
\hline \multicolumn{5}{|l|}{ Moradia } \\
\hline Casa & 80,73 & 88 & 92,59 & 125 \\
\hline Própria & 71,56 & 78 & 55,56 & 75 \\
\hline \multicolumn{5}{|l|}{ Número de cômodos } \\
\hline 2 a 3 cômodos & 21,1 & 23 & 20,74 & 28 \\
\hline 4 cômodos & 42,2 & 46 & 34,81 & 47 \\
\hline 5 cômodos ou mais & 36,7 & 40 & 42,96 & 37 \\
\hline
\end{tabular}




\begin{tabular}{lcccc}
\hline Estado nutricional pré-gestacional & & & & \\
\hline Baixo peso & 4,59 & 5 & 5,19 & 7 \\
\hline Adequado & 46,79 & 51 & 52,59 & 70 \\
\hline Sobrepeso & 33,94 & 37 & 27,41 & 38 \\
\hline Obesidade & 14,68 & 16 & 14,81 & 20 \\
\hline Estado nutricional final da gestação & & & & \\
\hline Baixo peso & 0,91 & 1 & 0 & 0 \\
\hline Adequado & 20,18 & 22 & 14,81 & 20 \\
\hline Sobrepeso & 36,69 & 40 & 37,77 & 51 \\
\hline Obesidade & 42,2 & 46 & 47,4 & 64 \\
\hline Pressão alta na gestação & 32,11 & 35 & 17,04 & 27 \\
\hline Esporte antes da gravidez & 43,12 & 47 & 28,89 & 39 \\
\hline Esporte durante a gravidez & 7,34 & 8 & 7,41 & 10 \\
\hline Fumo durante gravidez & 9,17 & 10 & 7,41 & 10 \\
\hline Drogas ilícitas antes da gravidez & 9,7 & 19 & 3,7 & 5 \\
\hline Drogas ilícitas durante gravidez & 8,26 & 9 & 3,7 & 5 \\
\hline Bebida alcoólica antes da gravidez & 29,36 & 32 & 22,96 & 31 \\
\hline Bebida alcoólica durante gravidez & 11,01 & 12 & 4,44 & 6
\end{tabular}

Tabela 2 - Resultados do teste Qui-quadrado. Parnamirim, RN, Brasil, 2015

\begin{tabular}{lc} 
Variáveis & p valor \\
\hline Idade $(<20$ anos e $>35$ anos) & $\leq 0,000032$ \\
\hline Obesidade e sobrepeso pré-gestacional & $\leq 0,012573$ \\
\hline Obesidade e sobrepeso no final da gestação & $\leq 0,006397$
\end{tabular}

No tocante às condições e características maternas, o sobrepeso e a obesidade antes e durante a gravidez são apontados como fator de risco para o nascimento do bebê prematuro (Tabela 2); houve aumento da prevalência de gestantes com índice de massa corporal com sobrepeso e obesidade durante a gestação, ademais, a maioria das mães mencionou sedentarismo desde a preconcepção (Tabela 1).

Por fim, na Tabela 1, identificou-se como estilos de vida deletérios à saúde materno-infantil o consumo de álcool, tabaco e drogas ilícitas durante a gestação. Logo, as mães com pressão alta durante a gravidez apresentaram predominância no estado nutricional com sobrepeso e obesidade.

$\mathrm{Na}$ Tabela 3, aponta-se que na nota de apgar do quinto minuto, alguns bebês prematuros apresentavam-se com asfixia, enquanto nenhum dos bebês a termo continuou com asfixia. Além disso, menos da metade dos bebês prematuros nasceu com o peso adequado e $11,93 \%(n=13)$ nasceram com peso extremamente baixo; enquanto nos bebês a termo, 91,11\% $(n=123)$ nasceram com o peso adequado. Por fim, 58,10\% ( $n=63)$ dos bebês prematuros nasceram com dificuldades de adaptar-se ao meio extrauterino ou com asfixia; ao passo que $96,30 \%(n=130)$ dos bebês a termo nasceram com bom estado de saúde. 
Tabela 3 - Características do nascimento de bebês prematuros e a termo. Parnamirim, RN, Brasil, 2015

\begin{tabular}{|c|c|c|c|c|}
\hline \multirow[t]{2}{*}{ Variáveis } & \multicolumn{2}{|c|}{ Pré-termo } & \multicolumn{2}{|c|}{ Termo } \\
\hline & $\%$ & $f$ & $\%$ & $f$ \\
\hline \multicolumn{5}{|l|}{ Sexo } \\
\hline Masculino & 54,13 & 59 & 40 & 54 \\
\hline Feminino & 45,87 & 50 & 60 & 81 \\
\hline \multicolumn{5}{|l|}{ Apgar 1' } \\
\hline$<5$ & 13,89 & 15 & 1,48 & 2 \\
\hline De 5 a 7 & 14,81 & 16 & 7,41 & 10 \\
\hline De 8 a 9 & 71,3 & 77 & 91,11 & 123 \\
\hline 10 & 0 & 0 & 0 & 0 \\
\hline \multicolumn{5}{|l|}{ Apgar 5' } \\
\hline$<5$ & 9,26 & 10 & 0 & 0 \\
\hline De 5 a 7 & 3,7 & 4 & 0 & 0 \\
\hline De 8 a 9 & 86,11 & 93 & 94,7 & 127 \\
\hline 10 & 0,93 & 1 & 5,93 & 8 \\
\hline \multicolumn{5}{|l|}{ Peso ao nascer } \\
\hline$<1000 \mathrm{~g}$ & 11,93 & 13 & 0 & 0 \\
\hline$<1500 \mathrm{~g}$ & 5,5 & 6 & 1,48 & 2 \\
\hline$<2500 \mathrm{~g}$ & 39,45 & 43 & 7,41 & 10 \\
\hline$\geq 2500 \mathrm{~g}$ & 43,12 & 47 & 91,11 & 123 \\
\hline \multicolumn{5}{|c|}{ Estado de saúde ao nascer } \\
\hline Bem de saúde & 41,9 & 46 & 96,3 & 130 \\
\hline Com problemas & 58,1 & 63 & 3,7 & 5 \\
\hline
\end{tabular}

Das mães com peso adequado ao final da gestação, apenas 35,29\% dos bebês prematuros tiveram peso adequado, ao nascer (Figura 1). Diferentemente de $97,62 \%$ dos bebês a termo nasceram com peso adequado (Figura 2).

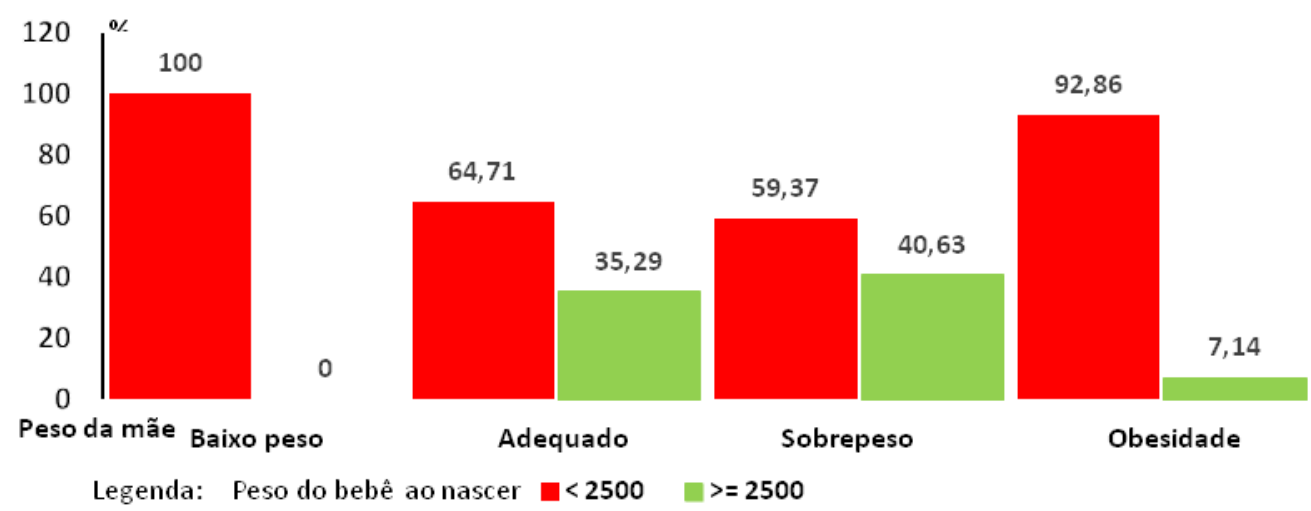

Figura 1 - Peso da mãe ao final da gestação X Peso ao nascer do bebê pré-termo. Parnamirim, RN, Brasil, 2015 


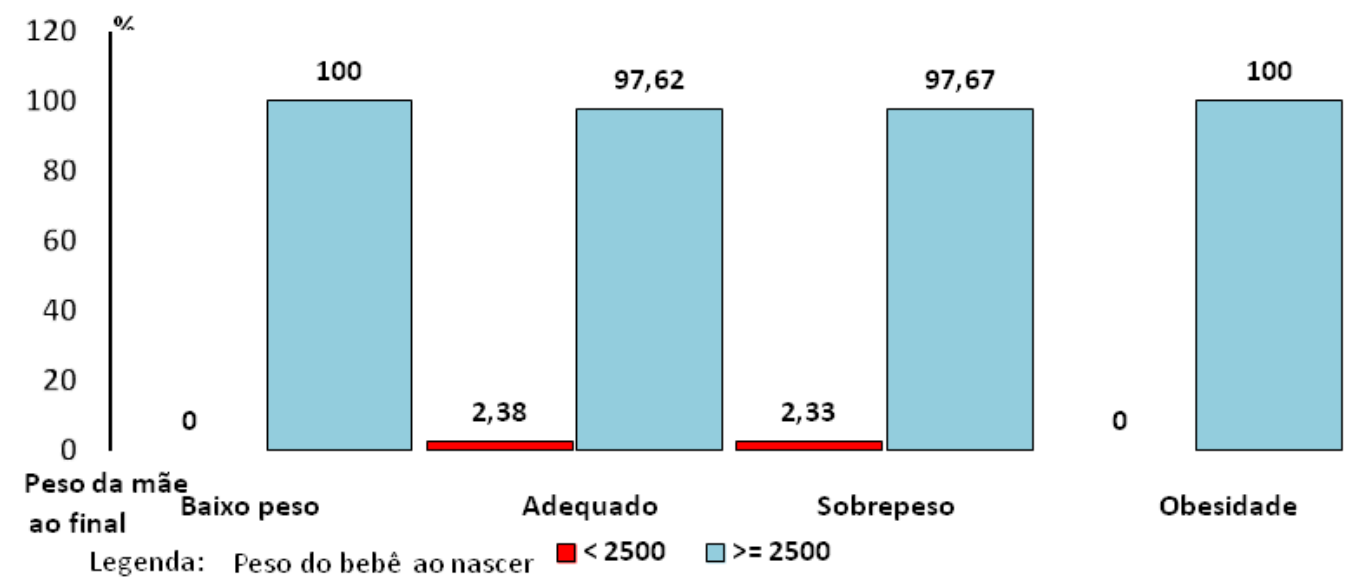

Figura 2 - Peso ao final da gestação X Peso ao nascer do bebê a termo. Parnamirim, RN, Brasil, 2015

Mães com baixo nível de escolaridade e baixa renda estão predispostas a alimentação inadequada. Dessa forma, a obesidade e sobrepeso, usuária de drogas ilícitas durante a gestação, e pressão arterial elevada podem indicar fator de risco ao nascimento do bebê prematuro, como evento desfavorável e de risco à saúde da criança.

\section{- DISCUSSÃO}

O perfil socioeconômico de mães de bebês prematuros e a termo apresentou prevalências semelhantes para baixo nível de escolaridade e renda. Em estudo de coorte realizado com diferentes grupos de renda, considera-se essa situação como fator de risco para o nascimento prematuro, pois dificulta o acesso à informação de saúde e à prestação de cuidado eficaz ${ }^{(17)}$.

Além disso, outros estudos apontam que a moradia inadequada (sem proteção para si própria e família, sem privacidade) e em casa alugada é também um fator de risco para o nascimento prematuro ${ }^{(17-19)}$. E como se pode observar ainda na Tabela 1, cerca de um quinto das mães de bebês prematuros e a termo reside em moradia com 2 a 3 cômodos e em moradia própria apenas pouco mais da metade das mães de bebês a termo.

Já em relação ao estado conjugal, a maioria das mães de bebês pré-termo e a termo referiu ser casada ou morar junto. Fator esse importante a ser ressaltado para que seja inclusa a paternidade em todo o ciclo gravídico puerperal, visando aos benefícios que a participação dos homens traz quando se mostra afetivo, aumentando a segurança da mulher e vínculo familiar consistente ${ }^{(20)}$.

A alimentação e o estado nutricional inadequado da gestante, antes e durante a gravidez, podem comprometer o desenvolvimento e crescimento fetal, bem como a evolução da gravidez ${ }^{(21)}$. Logo, estudos apontam que gestante com baixo peso e obesidade são fatores de risco para o nascimento prematuro ${ }^{(19,22)}$, com razão de chance de 3,030 para as gestantes obesas ${ }^{(23)}$.

Na Tabela 2, o sobrepeso e obesidade antes e durante a gestação apresentam-se como fator de risco para o nascimento do bebê prematuro, além disso, na Tabela 1, também se observa crescente número de mães com aumento de peso inadequado durante a gestação, divergindo do estudo realizado em um hospital universitário de São Paulo, em que as gestantes apresentaram-se eutróficas no ganho de peso ponderal em $45 \%$.

Destarte, a prática de exercícios físicos de intensidade moderada durante a gestação de risco habitual e inclusive antes da gestação pode ser benéfica para a mãe, visto que previne a obesidade, hipertensão arterial e diabetes gestacional ${ }^{(25)}$. Contudo, a Tabela 1 mostra que apenas cerca de um terço das mães em estudo praticava exercícios físicos antes da gravidez, acentuando ainda mais o sedentarismo durante a gestação. 
Uma revisão sistemática e metanálise referem que o consumo abusivo de álcool durante a gravidez aumenta os riscos de parto prematuro, então, deve-se estimular medidas de prevenção durante as consultas de pré-natal (26). Dessa forma, observa-se, na Tabela 1, que houve consumo de álcool pelas gestantes, todavia com redução no percentual do consumo entre o período antes e durante a gestação de $29,36 \%$ para $11,01 \%$ pelas mães de bebês prematuros e de $22,96 \%$ para $4,44 \%$ pelas mães de bebês a termo.

Outrossim, devem ser adotadas estratégias de intervenções baseadas na família e nas escolas visando à sensibilização de hábitos de vida saudável como o não uso de álcool, tabagismo e outras drogas, uma vez que o seu consumo são estilos de vida deletérios à saúde materno-infantil(27). Nesse sentido, a Tabela 1 apresenta consumo do tabaco pelas mães de bebês prematuros e a termo, e ainda mais agravante, o consumo de drogas ilícitas (maconha, crack) permaneceu antes e durante a gravidez praticamente com a mesma prevalência.

Uma das formas de avaliação das condições do recém-nascido foi o índice de Apgar. Na Tabela 3, aponta-se que alta prevalência dos bebês a termo nasceu sem asfixia; no entanto, dos bebês prematuros, $13,89 \%$ nasceram com asfixia grave e 14,81\% com asfixia moderada no primeiro minuto de vida, apresentando no quinto minuto melhoria na prevalência de toda classificação do Apgar, a qual prediz a assistência necessária para melhor adaptação do bebê ao meio extrauterino.

O peso ao nascer também é fator determinante do estado de saúde do recém-nascido, podendo ser influenciado por vários fatores, tais como baixo nível socioeconômico, nutrição inadequada, tabagismo, morbidade, durante a gestação. O estado nutricional materno tem efeito expressivo no crescimento e desenvolvimento fetal ${ }^{(28)}$.

Com isso, na Figura 2, observa-se que das mães com peso adequado ao final da gestação, a maioria das mães de bebês a termo pariu seus filhos com peso adequado. No entanto, as mães de bebês prétermo, apresentadas na Figura 1, apenas cerca de um terço dos bebês prematuros teve peso adequado ao nascer, sugerindo associação do baixo peso ao nascer com as morbidades apresentadas pelas mães durante a gestação e/ou decorrentes do nascimento antes do seu crescimento e desenvolvimento, conforme estudo em que a duração da gestação foi o principal motivo de evolução do peso ao nascer ${ }^{(29)}$.

Diante do exposto, cerca da metade dos bebês prematuros nasceu com problemas de saúde, enquanto praticamente todos os bebês a termos tiveram nascimento saudável, conforme apresentado na Tabela 4. Portanto, é imprescindível identificar os fatores de risco para o nascimento do bebê prematuro, visto que a maioria desses nascimentos pode ser evitada através de pré-natal de qualidade e acesso à educação em saúde. Além disso, ressalta-se a importância do planejamento familiar, pois através deste pode-se prestar orientações relacionadas à gravidez planejada para que os bebês nasçam com condições dignas de sobrevivência.

A presente pesquisa apresentou como limitações a dependência da memória do paciente quanto aos seus históricos de saúde antepassados e gestacional; e dos dados registrados nos prontuários e cartão das gestantes, por isso a importância da qualidade dos registros da assistência prestada no cotidiano. Então, para minimizá-las, buscou-se associar as informações referidas pelas mães e os dados registrados pelos profissionais. Salienta-se a necessidade de novos estudos com melhores níveis de evidências para causalidade do nascimento do bebê prematuro.

\section{CONCLUSÃO}

Mães com baixo nível de escolaridade, adolescente e baixa renda estão predispostas a alimentação inadequada e sedentarismo. Dessa forma, se observou durante a gestação, pressão arterial elevada; como também, a obesidade e sobrepeso da gestante apresentaram-se como fator de risco ao nascimento do bebê prematuro, fatores esses considerados como evento desfavorável e de risco à saúde da criança.

Diante desse perfil e condições maternas, houve importante prevalência de os bebês prematuros nascerem com dificuldades de adaptação ao meio extrauterino e dos bebês a termo nascerem saudáveis. Assim, os bebês prematuros nasceram com asfixia e baixo peso ao nascer, mesmo os bebês das mães 
com estado nutricional adequado, sugerindo associação com as morbidades maternas apresentadas durante a gestação.

Assim, é imprescindível a realização de planejamento familiar e fácil acesso aos métodos contraceptivos; educação em saúde para estimular hábitos de vida saudável desde a preconcepção; pré-natal de qualidade que possa diagnosticar precocemente alteração da pressão arterial, realizar sua monitorização e controle, como também diagnosticar em tempo oportuno as intercorrências na gestação.

\section{REFERÊNCIAS}

1. Goldenberg RL, Culhane JF, lams JD, Romero R. Epidemiology and causes of preterm birth. The Lancet. [Internet] 2008;371(9606) [acesso em 12 jan 2017]. Disponível: http://dx.doi.org/10.1016/S0140-6736(08)60074-4.

2. Assunção PL, Novaes HMD, Alencar GP, Melo ASO, Almeida MF. Fatores associados ao nascimento pré-termo em Campina Grande, Paraíba, Brasil: um estudo caso-controle. Cad. Saúde Pública. [Internet] 2012;28(6) [acesso em12 jan 2017]. Disponível: http://dx.doi.org/10.1590/S0102-311X2012000600007.

3. Central Intelligence Agency. The World Factbook. [Internet] 2014 [acesso em17 mar 2016]. Disponível: http:// www.indexmundi.com/g/r.aspx?c=us\&v=29\&l=pt.

4. Ministério da saúde (BR). Mortalidade infantil no Brasil: tendências, componentes e causas de morte no período de 2000 a 2010. Brasília (DF): Ministério da Saúde; 2011.

5. Black RE, Cousens S, Johnson HL, Lawn JE, Rudan I, Bassani DG, et al. Global, regional, and national causes child mortality in 2008: a systematic analysis. The Lancet. [Internet] 2010;375(9730) [acesso em 14 jan 2017]. Disponível: http://dx.doi.org/10.1016/S0140-6736(10)60549-1.

6. World Health Organization (WHO). Levels and trends in child mortality 2012. [Internet] Geneva: WHO; 2012 [acesso em 19 jan 2016]. Disponível: http://www.who.int/maternal_child_adolescent/documents/levels_trends_ child_mortality_2012/en/.

7. Kenner C, Boykova M. Enfermagem neonatal em um contexto global: principais realizações e progressos. Programa de Atualização em Enfermagem (PROENF)-Saúde Materna e Neonatal. 2014;5(2):9-46.

8. Coto FPG, Cruz FP, Mazier CZ. Factores asociados al parto prematuro en el departamento de Lempira, Honduras. Rev Med Hondur. [Internet] 2012;80(4) [acesso em 29 jan 2017. Disponível: http://www.bvs.hn/RMH/ pdf/2012/pdf/Vol80-4-2012-4.pdf.

9. Sampaio RMM, Pinto FJM, Sampaio JC. Fatores de risco associados à prematuridade em nascidos vivos no estado do Ceará. Rev. baiana saúde pública. [Internet] 2012;36(4) [acesso em 29 jan 2017]. Disponível: http://rbsp. sesab.ba.gov.br/index.php/rbsp/article/view/791/390.

10. Muglia LJ, Katz M. The enigma of spontaneous preterm birth. N Engl J Med. [Internet] 2010;362(6) [acesso em 29 jan 2017]. Disponível: http://dx.doi.org/10.1056/NEJMra0904308.

11. Almeida-filho N. Epidemiologia sem números. Rio de Janeiro: Campus; 1989.

12. Ministério da Saúde (BR). Lei n. 7498, de 25 de junho de 1986. Dispõe sobre a regulamentação do exercício profissional de enfermagem, e dá outras providências. Diário Oficial da União, Brasília, 26 jun. 1986. Seção 1.

13.Conselho Federal de Enfermagem. Resolução COFEN n. 0478/2015. Normatiza a atuação e a responsabilidade civil do Enfermeiro Obstetra e Obstetriz nos Centros de Parto Normal e/ou Casa de Parto e dá outras providências. Brasília: COFEN; 2015.

14. Ministério da Saúde (BR). DATASUS - Informações de Saúde. Estatísticas vitais. Mortalidade infantil: desde 1996. [Internet] Brasília (DF): Ministério da Saúde; 2012 [acesso em 12 jan 2017]. Disponível: http://www2.datasus. gov.br/DATASUS/index.php?area=0205\&id=6938\&VObj=http://tabnet.datasus.gov.br/cgi/deftohtm.exe?sim/cnv/ inf10.

15. Ministério da Saúde (BR). Secretaria de Atenção à Saúde. Departamento de Atenção Básica.Atenção ao pré- 
natal de baixo risco. Brasília (DF): Ministério da Saúde: 2012.

16. World Health Organization (WHO). Public health aspects of low birth weight: third report of the Expert Committee on Maternal and Child Health. [Internet] Geneva: WHO; 1961 [acessoem12 fev2017]. Disponível: http://apps.who.int/iris/handle/10665/40487.

17. Silva AMR, de Almeida MF, Matsuo T, Soares DA. Fatores de risco para nascimentos pré-termo em Londrina, Paraná, Brasil. Cad. Saúde Pública. [Internet] 2009;25(10) [acesso em 30 jan 2017]. Disponível: http://dx.doi. org/10.1590/S0102-311X2009001000004.

18. Silveira MF, Victora CG, Barros AJD, Santos IS, Matijasevich A, Barros FC. Determinantes de nascimento pré-termo na coorte de nascimentos de 2004, Pelotas, Rio Grande do Sul, Brasil. Cad. Saúde Pública. [Internet] 2010;26(1) [acesso em 30 jan 2017]. Disponível: http://dx.doi.org/10.1590/S0102-311X2010000100019.

19. Niedhammer I, Murrin C, O'Mahony D, Daly S, Morrison JJ, Kelleher CC, et al. Explanations for social inequalities in preterm delivery in the prospective Life ways cohort in the Republic of Ireland. Eur J Public Health. [Internet] 2012;22(4) [acesso em 30 jan 2017]. Disponível: https://doi.org/10.1093/eurpub/ckr089.

20. Petito ADC, Cândido ACF, Ribeiro LO, Petito G. A importância da participação do pai no ciclo gravídico puerperal: uma revisão bibliográfica. REFACER. [Internet] 2015;4(1) [acesso em 02 fev 2017]. Disponível: http:// ceres.facer.edu.br/revista/index.php/refacer/article/view/70.

21. Siega-Riz AM, Viswanathan M, Moos MK, Deierlein A, Mumford S, Knaack J, et al. A systematic review of outcomes of maternal weight gain according to the Institute of Medicine recommendations: birth weight, fetal growth, and postpartum weight retention. Am J Obstet Gynecol. [Internet] 2009;201(4) [acesso em 02 fev 2017]. Disponível: http://dx.doi.org/10.1016/j.ajog.2009.07.002.

22. Chen A, Klebanoff MA, Basso O. Pre-pregnancy body mass index change between pregnancies and preterm birth in the following pregnancy. Paediatr Perinat Epidemiol. [Internet] 2009;23(3) [acesso em 02 fev 2017 ]. Disponível:http://dx.doi.org/10.1111/j.1365-3016.2009.01029.x.

23. Zhang YP, Liu XH, Gao SH, Wang JM, Gu YS, Zhang JY, et al. Risk factors for preterm birth in five Maternal and Child Health hospitals in Beijing. PLoS One. [Internet] 2012;7(12) [acesso em 02 fev 2017]. Disponível: http:// dx.doi.org/10.1371/journal.pone.0052780.

24. Fazio ES, Nomura RMY, Dias MCG, Zugaib M. Consumo dietético de gestantes e ganho ponderal materno após aconselhamento nutricional. Rev. Bras. Ginecol. Obstet. [Internet] 2011;33(2) [acesso em 12 fev 2017 ]. Disponível: http://www.scielo.br/pdf/rbgo/v33n2/v33n2a06.pdf.

25. Zavorsky GS, Longo LD. Exercise guidelines in pregnancy. Sports Med. [Internet] 2011;41(5) [acesso em 12 fev2017]. Disponível: http://dx.doi.org/10.2165/11583930-000000000-00000.

26. Patra J, Bakker R, Irving H, Jaddoe VWV, Malini S, RehmJ. Dose-response relationship between alcohol consumption before and during pregnancy and the risks of low birth weight, preterm birth and small-size-forgestational age (SGA) - A systematic review and meta-analyses. BJOG. [Internet] 2011;118(12) [acesso em 12 fev 2017]. Disponível: http://dx.doi.org/10.1111/j.1471-0528.2011.03050.x.

27. Balázs P, Rákóczi I, Grenczer A, Foley KL. Risk factors of preterm birth and low birth weight babies among Roma and non-Roma mothers: a population-based study. Eur J Public Health. [Internet] 2013;23(3) [acesso em 12 fev 2017]. Disponível: http://dx.doi.org/10.1093/eurpub/cks089.

28. dos Santos MTM, Campos T, Silva ACP, Andrade BD, Cândido APC, Oliveira RMS, et al. Fatores relacionados ao peso ao nascer: influência de dados gestacionais. Rev. Méd. Minas Gerais. [Internet] 2015;25(2) [acesso em 14 fev 2017]. Disponível: http://www.dx.doi.org/10.5935/2238-3182.20150037.

29. Cristóvão FS, Pedraza DF. Tendência secular do peso ao nascer nas cidades de Cabedelo e Cacimbas, nordeste do brasil. Revista Espaço Para a Saúde. [Internet] 2015;16(1) [acesso em 14 fev 2017]. Disponível: http://dx.doi. org/10.22421/1517-7130.2015v16n1p7. 